\title{
Measuring quality of life after radical prostatectomy from the patient's perspective
}

\author{
Joseph L. Chin, MD, FRCSC
}

See related article on page 445

$\mathrm{n}$ recent years, there have been an increasing number of reports in the urologic literature on health-related quality of life (HRQoL) issues, such as the comparison of HRQoL in various forms of urinary diversion after cystectomy for bladder cancer and, more recently, the effects of radical prostatectomy on HRQoL and comparison of HRQoL of various treatment modalities for prostate cancer. Most of these reports deal with specific domains affected by the treatment instead of the effects on the overall patient wellbeing or "global HRQoL," as gauged by the patient's utility. Moreover, the studies often tend to report more of a snapshot at a given time after therapy, rather than a longitudinal chronicling of changes in the various domains.

Urologists often refer to the post-radical prostatectomy "Holy Grail" or "Tri-fecta" of biochemical disease-free status, perfect urinary continence and recovery of sexual function; each of these 3 parameters acts as a binary function, essentially from the health-provider's viewpoint. In this issue, $\mathrm{Ku}$ and colleagues ${ }^{1}$ make an important contribution to the uro-oncologic literature with their utility-based report before radical prostatectomy and several subsequent time points; they analyze several domains from the patient's own viewpoint and individual "value scale." Not surprisingly, the symptom scores and global health utility status dropped markedly in the early postoperative recuperation period. A noteworthy finding was the persistence, to a lesser extent, of these health status deficits well beyond the "physical recovery" period.

These findings are relevant and important in counselling patients on management options and in ensuring that patients do not have unrealistic postoperative expectations, especially those patients with low-risk disease where overtreatment is a possibility. It would be of interest to compare the results in patients undergoing external beam radiotherapy, ${ }^{2,3}$ and to duplicate the study in patients undergoing minimally invasive forms of intervention, such as brachytherapy, cryoablation and high-intensity focused ultrasound (HIFU). The results of these studies would be useful in treatment decisions. Also of interest would be similar longitu- dinal studies conducted in jurisdictions with different cultural backgrounds and different ethnic composition.

From the Departments of Surgery \& Oncology, Divisions of Urology \& Surgical Oncology, London Health Sciences Centre-Victoria Hospital, London, ON

Competing interests: None declared.

This paper has been peer-reviewed.

\section{References}

1. Ku J, Krahn M, Trachtenberg J, et al. Changes in health utilities and health-related quality of life over 12 months following radical prostatectomy. Can Urol Assoc J 2009;3:445-52.

2. Waldmann A, Rohde V, Bremner K, et al. Measuring prostate-specific quality of life in prostate cancer patients scheduled for radiotherapy or radical prostatectomy and reference men in Germany and Canada using the Patient Oriented Prostate Utility Scale-Psychometric (PORPUS-P). BMC Cancer 2009;23:295.

3. Krahn MD, Bremner KE, Tomlinson G, et al. Utility and health-related quality of life in prostate cancer patients 12 months after radical prostatectomy or radiation therapy. Prostate Cancer Prostatic Dis 2009;12:361-8.

Correspondence: Dr. Joseph Chin, Departments of Surgery \& Oncology, Divisions of Urology \& Surgical Oncology, University of Western Ontario, London Health Sciences Centre-Victoria Hospital, 800 Commissioners Road East, Suite (3-120, London, ON N6A 4G5; fax: 519-685-8450; joseph.chin@|hsc.on.ca 